\title{
THRESHOLD VOLTAGE VARIATIONS IN N-CHANNEL MOS TRANSISTORS AND MOSFET-BASED SENSORS DUE TO OPTICAL RADIATION
}

\author{
W WLODARSKI, P BERGVELD and J A VOORTHUYZEN
}

Department of Electrical Engineering, Twente Universtty of Technology, $P O$ Box 217, 7500 AE Enschede (The Netherlands)

(Recelved November 29 1985, in revised form February 21, 1986, accepted May 15, 1986)

\section{Abstract}

The influence of optical radiation on the MOSFET threshold voltage has been investıgated theoretically as well as experımentally For conventional MOSFETs the influence is negligible, but for open-gate FET-based sensors, such as the ISFET, optical radiation can cause a considerable threshold voltage shift An explanation of the threshold voltage shift due to illumination is given, based on the analysis of quasi-equilibrium effects in an illuminated semiconductor surface layer The relation between the threshold voltage and the optical radiation intensity has been derived Experimental results are given

\section{Introduction}

In recent years, there has been a growing interest in the utilization of insulated-gate field-effect transistor structures and related devices as sensors For example, visible and infrared imagers, piezoelectric strain sensors, various chemical sensors, as well as humidity and gas sensors based on these structures have all been reported in the literature

Although there is a vast store of knowledge concerning the effect of optical radiation on the electrophysical parameters of MIS capacitors [1 - 4], no information is avallable pertaining to the effect of optical radiation on MISFET parameters and characteristics, which is a serious drawback with respect to the application of illuminated MOSFET-based sensors For conventional MISFETs this problem is of minor importance, but for open-gate FET-based sensors it is essential to consider it

For instance, we observed a gate-source voltage shift of approximately $50 \mathrm{mV}$ in ISFETs applied in a source follower concept, due to normal ambient lighting This voltage shift is comparable to that produced by a change of $1 \mathrm{pH}$ unit in a test solution, and indicates the importance of effectively reducing the ISFET light sensitivity 
Recording an $I_{\mathrm{d}}-V_{\mathrm{gs}}$ curve, we observed a drain-current shift due to llumination that is caused by two effects drain-bulk diode photocurrent (vertical shift) and threshold voltage shift (horizontal) Measurements have shown that the electron Hall moblity is independent of light up to an intensity of $\sim 100 \mu \mathrm{W} / \mathrm{mm}^{2}$, and therefore we assume the drift mobility is also independent of light The nature of the drain-bulk photocurrent is well known from ordinary photodıodes, therefore our current research efforts have been concentrated on the MOSFET threshold voltage shift as a function of optical radiation

\section{Theory}

The threshold voltage $V_{\mathrm{t}}$ of an N-channel MOS transistor can be written as follows

$$
V_{\mathrm{t}}=\phi_{\mathrm{MS}}-\frac{Q_{\mathrm{ss}}}{C_{\mathrm{ox}}}+2 \phi_{\mathrm{F}}-\frac{Q_{\mathrm{B}}}{C_{\mathrm{ox}}}=V_{\mathrm{f}}+2 \phi_{\mathrm{F}}+\frac{+2 \sqrt{\left|\epsilon q N \phi_{\mathrm{F}}\right|}}{C_{\mathrm{ox}}}
$$

The symbols used are defined as follows

$\phi_{\mathrm{F}}=$ Fermi potential,

$\phi_{\mathrm{MS}}=$ metal-semiconductor Fermi potential difference,

$\mathrm{Q}_{\mathrm{ss}}=$ extrinsic charge due to surface states, interface energy states, oxide traps, etc ,

$\mathrm{C}_{\mathrm{ox}}=$ gate oxide capacitance per unit area, face,

$Q_{B}=$ charge per unit area in the depletion region underneath the sur-

$V_{f}=$ flat band voltage,

$\mathbf{N}=$ impurity concentration,

$\epsilon=$ permittivity of the semiconductor

To express this equation as a function of the optical radiation intensity $I_{\mathrm{OR}}$, each of the vanables must be written explicitly in terms of $I_{\mathrm{OR}}$

When optical radiation acts on the MOSFET, the equilibrium conditions in a semiconductor are disturbed by light-induced excessive electronhole pairs The incident light causes a perturbation of the carrier concentrations throughout the semiconductor, producing in turn separate hole and electron quasi-Fermı levels

When describing the phenomena occurring in an illuminated MOSFET, the following simplifying assumptions have been made

(1) The considered structure is assumed to satisfy the conditions of the 'ideal' MOS structure

(2) The bipolar generation occurs uniformly within the whole volume of silicon under the gate

(3) Quasi-Fermı levels are spatially constant and the positions of these levels are fixed by the light-perturbated carrier concentration This assumption is acceptable in the case of weak surface recombination [5] 
(4) The metal-semiconductor Fermi potential difference $\phi_{M S}$ as well as the surface state density $Q_{\mathrm{ss}}$ are assumed to be independent of optical radiation

(5) Surface recombination is ignored

(6) The lifetimes of electrons and holes are equal to each other and constant over the whole volume and surface layer of the scmiconductor

(7) Optically stimulated charge exchange with surface states as well as photoemission to the dielectric are neglected

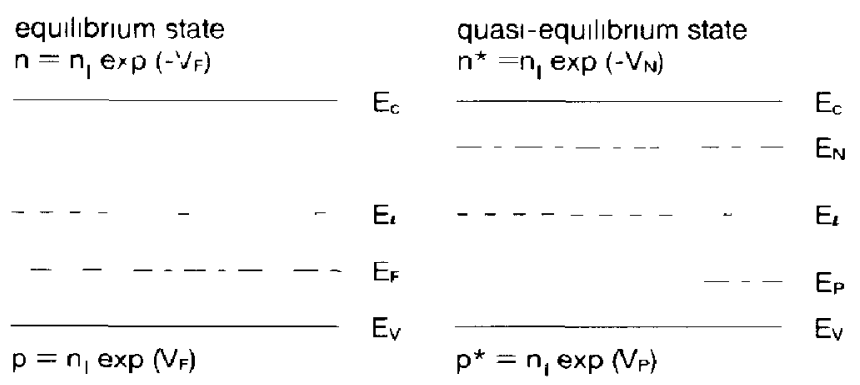

Fig 1 Energy diagrams of a semiconductor for equilibrium and quasi-equilibrium states $E_{\mathrm{v}}=$ energy of valence band edge, $E_{\mathrm{c}}=$ energy of conduction band edge, $E_{1}=$ energy of the middle forbidden gap, $E_{\mathrm{F}}=$ energy of the Fermi level, $E_{\mathrm{N}}=$ energy of the electron quası-Fermı level, $E_{\mathrm{P}}=$ energy of the hole quası-Fermı level

The energy diagrams of a semiconductor are now considered (Fig 1) The thermodynamic equilibrium condition

$n p=n_{1} \exp \left(-V_{\mathrm{F}}\right) n_{1} \exp \left(V_{\mathrm{F}}\right)$

with $V_{\mathrm{F}}$ the normalized Fermi potential $\left(V_{\mathrm{F}}=\phi_{\mathrm{F}} q / k T\right)$ and $n_{1}$ the instrinsic concentration, is disturbed by optical radiation producing separate hole and electron quasi-Fermı levels In the normalized form these are respectively denoted as $V_{\mathrm{P}}$ and $V_{\mathrm{N}}$, and can be written as

$$
\begin{gathered}
V_{\mathrm{N}}=-\frac{E_{\mathrm{N}}-\mathrm{E}_{1}}{k T} \\
V_{\mathrm{P}}=-\frac{E_{\mathrm{P}}-E_{1}}{k T}
\end{gathered}
$$

For a low light generation level it is possible to assume that the temperature and energy of the crystal do not change This state is the so called quasiequilibrium state The total electron and hole quasi-equilibrium concentrations can then be described by the following equations

$n^{*}=n+\Delta n$

$p^{*}=p+\Delta p$

where $n^{*}$ and $p^{*}$ are the electron and hole quasi-equilibrium concentrations, respectively (all quantities for quasi-equilibrium states will be signified by asterisks) 
The product of the electron and hole concentrations for the quasiequilibrium state differs from that for the equilibrium state

$n^{*} p^{*}=n_{1} \exp \left(-V_{\mathrm{N}}\right) n_{1} \exp \left(V_{\mathrm{P}}\right)=n_{1}{ }^{2} \exp \left(V_{\mathrm{P}}-V_{\mathrm{N}}\right)$

The distance between the quasi-Fermı levels $V_{P}$ and $V_{N}$ characterizes the deviation from the equilibrium state

The product of $n^{*}$ and $p^{*}$ can now also be written as

$n^{*} p^{*}=n_{1}^{* 2}$

where $n_{1}{ }^{*}$ is equivalent to the value of $n_{1}$ under illuminated conditions, which we will call the effective intrinsic concentration

It can be shown that the description of surface region parameters in the quasi-equilibrium state can be presented analogously to that of the equilibrium state, replacing the instrinsic concentration $n_{2}$ by the effective instrinsic concentration $n_{1}{ }^{*}$ and the normalized Fermi potential $V_{F}=\phi_{F} q / k T$ by the normalized effective quasi-equilibrium Fermı potential $V_{\mathrm{F}}{ }^{*}$ We thus obtain

$n^{*}=n_{\mathrm{x}}{ }^{*} \exp \left(-V_{\mathrm{F}}{ }^{*}\right)$

$p^{*}=n_{1}^{*} \exp \left(V_{\mathrm{F}}^{*}\right)$

where

$$
V_{\mathrm{F}}^{*}=\frac{V_{\mathrm{P}}+V_{\mathrm{N}}}{2}
$$

is the so-called quasi-equilibrium effective Fermi potential

The main purpose of the present description is to analyse optical radiation effects on the MOSFET threshold voltage In order to achieve this purpose, we first have to combine quasi-equilıbrium parameters $n_{1}{ }^{*}$ and $V_{\mathrm{F}}{ }^{*}$ with the light excitation factor $R$, which is usually defined as [3]

$R=\frac{\Delta n}{n_{1}}=\frac{\Delta p}{n_{1}}$

Substituting eqn (12) into eqns (5) and (6), and realizing that $n=$ $n_{1} \exp \left(-V_{\mathrm{F}}\right), p=n_{1} \exp \left(V_{\mathrm{F}}\right), n^{*}=n_{1} \exp \left(-V_{\mathrm{N}}\right), p^{*}=n_{1} \exp \left(V_{\mathrm{P}}\right)$, as indicated in equations (2) and (7) respectively, the electron and hole quasi-Fermı levels are therefore given by

$V_{\mathrm{N}}=-\ln \left[R+\exp \left(-V_{\mathrm{F}}\right)\right]$

$V_{\mathrm{P}}=\ln \left[R+\exp \left(V_{\mathrm{F}}\right)\right]$

Substitution of these expressions into eqn (11) results in an effective quasi-equilibrium Fermı potential having the following form

$V_{F}^{*}=\frac{1}{2} \ln \left[\frac{R+\exp \left(V_{\mathrm{F}}\right)}{R+\exp \left(-V_{\mathrm{F}}\right)}\right]$

Substitution of eqns (13) and (14) into eqn (7) results in an effective intrinsic quasi-equilibrium concentration of the following form 
$n_{1}{ }^{*}=n_{1}\left\{\left[R+\exp \left(V_{\mathrm{F}}\right)\right]\left[R+\exp \left(-V_{\mathrm{F}}\right)\right]\right\}^{1 / 2}$

It follows from relations (15) and (16) that as the generation level grows, the effective Fermi level approaches the middle of the energy gap and the effective intrinsic concentration increases

The effective quasi-equilibrium Fermi potential is plotted in Fig 2 as a function of the light excitation factor

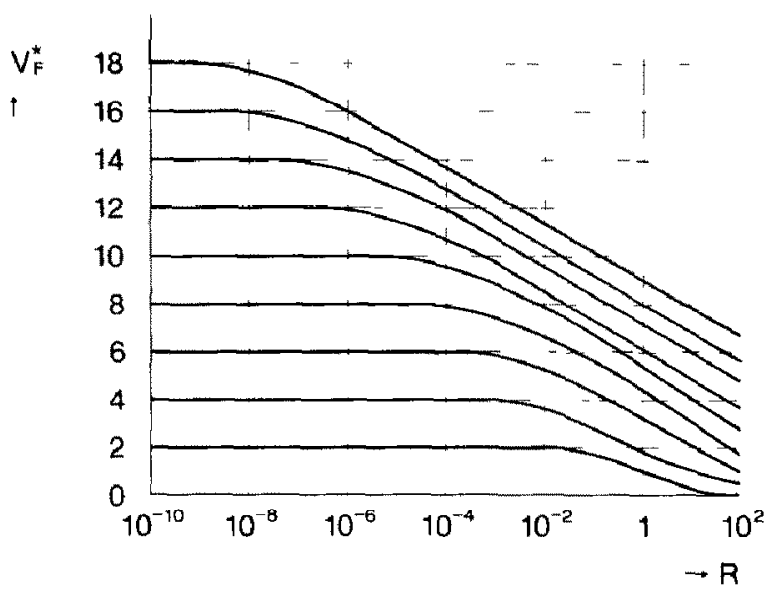

Fig 2 Normalized effective Fermi potential, $V_{\mathrm{F}}{ }^{*}$, us light excitation factor $R$, with the normalızed Fermı potential $V_{\mathrm{F}}$ in a non illuminated semiconductor as a parameter Note that $V_{\mathrm{F}}=18$ for the upper curve etc, for $V_{\mathrm{F}}{ }^{*}=V_{\mathrm{F}}$ if $R$ becomes zero

Again considering eqn (1), we see that the threshold voltage $V_{t}$ depends on the light excitation factor through the Fermi potential

The equation for the effective threshold voltage $V_{t}^{*}$ is

$V_{\mathrm{t}}^{*}=V_{\mathrm{f}}+\frac{2 k T}{q} V_{\mathrm{F}}^{*}+\frac{2\left(\epsilon N k T\left|V_{\mathrm{F}}^{*}\right|\right)^{1 / 2}}{C_{\mathrm{ox}}}$

Substitution of eqn (15) into eqn (17) results in an expression for the effective threshold voltage $V_{\mathrm{t}}{ }^{*}$ as a function of $R$,

$$
\begin{aligned}
V_{\mathrm{t}}^{*}= & V_{\mathrm{f}}+A_{1}\left\{\ln \left[R+\exp \left(V_{\mathrm{F}}\right)\right]-\ln \left[R+\exp \left(-V_{\mathrm{F}}\right)\right]\right\}+A_{2}\left\{\ln \left[R+\exp \left(V_{\mathrm{F}}\right)\right]\right. \\
& \left.-\ln \left[R+\exp \left(-V_{\mathrm{F}}\right)\right]\right\}^{1 / 2}
\end{aligned}
$$

where

$$
A_{1}=k T / q, \quad A_{2}=(2 \epsilon N k T)^{1 / 2} / C_{\circ \mathrm{x}}
$$

Figure 3 shows the theoretical effective threshold voltage $V_{t}^{*}$ as a function of the light-excitation factor for one of the investigated devices 


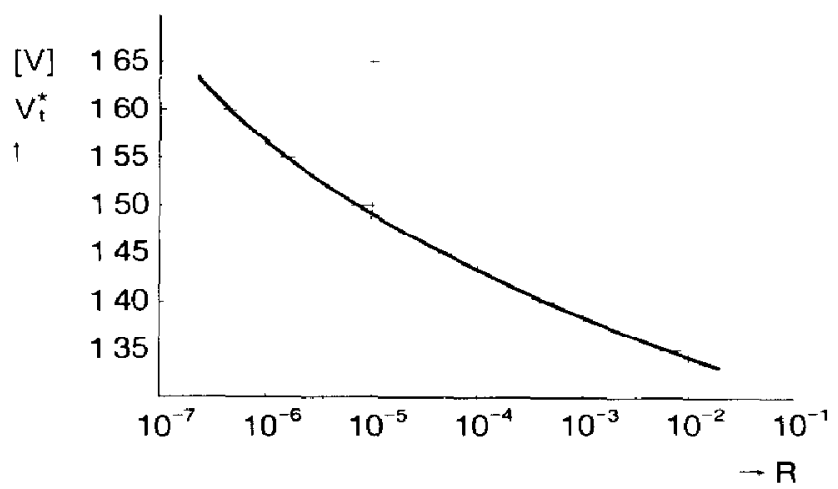

Fig 3 Calculated effective threshold voltage $V_{t} *$ as a function of light excitation factor $R T=300 \mathrm{~K}$

\section{Experumental}

In order to verify the theoretical considerations given in Section 2 with practical experiments using MOSFETs under illumination, we need a relation that links the light excitation factor $R$ with such experimental variables as optical radiation intensity, light wavelength etc

The light excitation factor $R$ is not directly measurable, but could be accurately determined from the light-frequency $C-V$ data [3] Based on Frankl and Ulmer's theory [4,6], Pierret and Sah [3] have found the following for $\mathrm{MOS} \mathrm{S}_{1}-\mathrm{SiO}_{2}$ structures

$R \simeq\left[\frac{\tau_{\mathrm{no}}}{n_{1} h \nu} \frac{\alpha}{1+\alpha L_{\mathrm{n}}}\right] I_{\mathrm{OR}}=k_{1} I_{\mathrm{OR}}$

where

$\tau_{\text {no }}=$ semiconductor minonty carrier lifetime,

$\alpha=$ semiconductor absorption coefficient,

$\mathrm{L}_{\mathrm{n}}=$ minority carrier diffusion length $\left(L_{\mathrm{n}}=\left(D_{\mathrm{n}} \tau_{\mathrm{no}}\right)^{1 / 2}\right.$ where $D_{\mathrm{n}}$ is the diffusion coefficient),

$\nu=$ frequency of the incident light,

$I_{\mathrm{OR}}=$ intensity of the optical radiation at the point of entry into the semiconductor

However, Pierret and Sah [3] also found that the light excitation factor $R$ is not exactly proportional to $I_{\mathrm{OR}}$ but that it is almost proportional to $I_{\mathrm{OR}}{ }^{15}$, which means a significant deviation from the expected linear dependence

In our experiments we have used n-channel normally-off MOSFETs We used a standard LOCMOS technology with ion implantation facilities and (100) oriented wafers Other process parameters are stopper implantation $N_{\mathrm{A}}=5 \times 10^{16} \mathrm{~cm}^{-3}$, gate-oxide thickness $d_{\mathrm{ox}}=75 \mathrm{~nm}$, polysilicon gate thickness $d=05 \mu \mathrm{m}$, threshold voltage $\approx+17 \mathrm{~V}$ 
As light sources we used a helium-neon laser $(\lambda=063 \mu \mathrm{m})$ and a socalled 'cold-light source' type KL 150B from Schott-Main (F R G) The light-meter was a fibre optics multimeter, model 22 LA produced by Photodyne Inc (U S A ) Figure 4 shows experimental values of the threshold voltage $V_{t}{ }^{*}$ as a function of optical radiation intensity $I_{O R}$ for the investigated MOSFET, of which the theoretical effective threshold voltage relation as a function of light excitation factor $R$ is shown in Fig 3 It can be concluded that the shapes of both the theoretical and experimental curves are in good agreement with each other

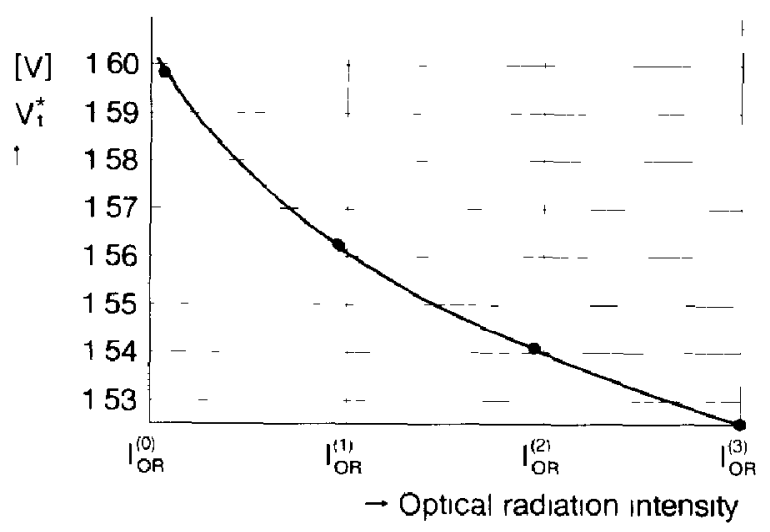

Fig 4 Experimental values of threshold voltage $V_{\mathrm{t}} *$ as a function of light intensity $I_{\mathrm{OR}}$ (linear scale) Intensity $I_{\mathrm{OR}}^{(3)}$ corresponds to approximately $04 \mu \mathrm{W} / \mathrm{mm}^{2} T=300 \mathrm{~K}$, $V_{\mathrm{d}}=05 \mathrm{~V}$

\section{Conclusions and discussion}

Using monochromatic illumination, the threshold voltage photoresponse of n-channel MOSFETs has been investigated and compared to the theoretical predictions

In general, the theory has been found to describe qualitatively the observed threshold voltage modulation of the MOSFET device due to illumination Agreement between theory and experiment, however, is only qualitative Quantitative agreement between theory and experiment is impaired by the fact that the constant $k_{1}$, as introduced in eqn (20), is not known

Additionally, it should be noted that this constant $k_{1}$ depends strongly on the properties of the sllicon, which impairs the possibility of comparing our results with those of Pierret and Sah [3], for example

We believe that by using MOSFET structures under monochromatic illumination, we can carefully estimate the light excitation factor $R$ by measuring the threshold voltage shift

In our experments we also used ISFETs with a sandwich dielectric of $\mathrm{SiO}_{2}$ and $\mathrm{Al}_{2} \mathrm{O}_{3}$ or $\mathrm{SiO}_{2}$ and $\mathrm{Ta}_{2} \mathrm{O}_{5}$ Therr behaviour under illuminated cond1- 
tions was similar to that of the MOSFET Therefore we conclude that the theoretical procedure proposed here may be useful in the analysis of the threshold voltage of all MOSFET-based sensors under lllumination

\section{References}

$1 \mathrm{~J}$ Grosvalet and C Jund, Influence of illumination on MIS capacitance in the strong inversion region, IEEE Trans Electron Devices, ED 14 (1967) 777780

2 A S Grove, B E Deal, E H Snow and C T Sah, Investigation of thermally oxidized silicon surfaces using metal-oxide semiconductor surfaces, Solid-State Electron, 8 (1965) $145-163$

3 R $\quad F$ Pierret and C $T$ Sah, Quantitative analysis of the effects of steady-state il lumination of the MOS capacitor Solld State Electron, 13 (1970), part 1, 269 288, part II, $289-302$

$4 \mathrm{D} R$ Frankl and $\mathrm{E}$ A Ulmer, Theory of the small signal photovoltage at the semconductor surface, Surface Scl, 6 (1966) 115

5 A Many, Y Goldstein and N B Grover, Semiconductor Surfaces, North Holland, Amsterdam, 1965

6 D Rrankl, Conditions for quasi-equilbrium in semiconductor space-charge layers, Surface Sct, 3 (1965) 101

\section{Biographies}

Johannes Arle Voorthuyzen was born in Capelle a/d IJssel, The Netherlands, on April 22, 1959 He recieved the M S degree in electrical eng1neering from the Delft University of Technology, Delft, The Netherlands, in 1982 In the same year he joined the Bio-Information Group, Department of Electrical Engineering, Twente University of Technology, where he is working towards his $\mathrm{Ph} \mathrm{D}$ degree His current research is focused on electromechanical sensors for biomedical applications

Plet Bergueld was born in Oosterwolde, The Netherlands, on January 26, 1940 He received the M S degree in electrical engineering (electronics) from Eindhoven University of Technology, Emdhoven, The Netherlands, in 1965, and the $\mathrm{Ph} \mathrm{D}$ degree from Twente University of Technology, Enschede, The Netherlands, in 1973

Since 1965 he has been a member of the Bio-information Group, Department of Electrical Engmeering, Twente University of Technology He is also member of the Coordination Centre for Biomedical Engineering and a member of the Research Unit 'Sensors and Actuators' of this University In 1981 he became a member of the Semiconductor Group of the Foundation for Fundamental Research on Matter in The Netherlands

The subject of his dissertation was the ion-sensitive field-effect transistor (ISFET) and the OSFET He is involved in research on electronic measuring and stumulating methods in physiological systems, with special attention on in vivo biosensors He lectures on biomedical instrumentation to 
graduate students of Twente University of Technology and medical personnel of affiliated hospitals

In 1984 he was appointed as full professor in biosensor technology at the Twente University of Technology

Wojclech Wlodarskl recelved the $\mathrm{M} \mathrm{Sc}$ and $\mathrm{Ph} \mathrm{D}$ degrees in electronics from the Warsaw Technical University, Warsaw, Poland in 1962 and 1971 respectively

In 1983 he was appointed as full professor in electronics at the Warsaw Technical University

His research includes solid state device theory and fabrication, especially for sensor applications, and electronic instrumentation

During 1984 and 1985 he worked at the Twente University of Technology, Enschede, The Netherlands and at the University of Toronto, Toronto, Canada on the subject of temperature and light compensation methods in con-sensitive-field-effect transistors and computer modelling of ISFET performance

He is currently a visiting professor in the Department of Bioengineering at the University of Utah, Salt Lake City, U S A His present interest is solid state chemical sensors 